\title{
CLONING AND CHARACTERIZATION OF A WHEAT RING FINGER GENE TaRHA2b WHOSE EXPRESSION IS UP- REGULATED BY ABA TREATMENT
}

\author{
LI, D. B. - LYU, G. Z. - LYU, J. P. - NIU, H. B. - WANG, X. - YIN, J. \\ National Engineering Research Center for Wheat, State Key Laboratory of Wheat and Maize \\ Crop Science, Collaborative Innovation Center of Henan Grain Crop \\ Henan Agricultural University, Zhengzhou 450002, China \\ ${ }^{*}$ Corresponding author \\ e-mail:xmzxyj@126.com; phone: +86-03-716-355-8203 \\ (Received 27 $7^{\text {th }}$ Feb 2019; accepted $3^{\text {rd }}$ May 2019)
}

\begin{abstract}
Preharvest sprouting in wheat (Triticum aestivum L.) is the germination of grains in the ears when long range rainfall or damp conditions prior to harvest occur. E3 ubiquitination (type RING-H2) RING finger protein plays a key role in dealing with abiotic stresses in plants, which could be used to improve the PHS resistance of wheat. The full-length cDNA of a wheat RING finger gene named $T a R H A 2 b$ was firstly cloned from wheat. Bioinformatic analysis and expression profile analysis of the TaRHA $2 b$ gene were carried out. The results indicated that the full-length cDNA of TaRHA2b was $845 \mathrm{bp}$ containing $465 \mathrm{bp}$ open reading frame which encoded 154 amino acid residues. The genomic TaRHA2b gene had no introns. The protein encoded by TaRHA2b gene was consisted of Zinc finger RING-type profile. Blast and phylogenetic analysis showed that the protein encoded by TaRHA2b shared the identity with RHA2b from the Arabidopsis. The result of semi RT-PCR showed that expression of TaRHA2b gene was significantly tissue-specific. The result of qRT-PCR showed that the expression of TaRHA2b in the seeds was significantly higher than the expression of gene expression after soaking germination. The sensitivity of this gene to abscisic acid was significantly increased. TaRHA2b gene may play an important role in seed dormancy during germination, which could be used to improve the PHS resistance of wheat.

Keywords: wheat, bioinformatics analysis, expression profile, seed dormancy, preharvest sprouting
\end{abstract}

Abbreviations: ABA, abscisic acid; PHS, preharvest sprouting; RING, Really Interesting New Gene; E1, ubiquitin-activating enzymes; E2, ubiquitin-conjugating enzymes; E3, ubiquitin protein ligase; ORF, open reading frame; Rel.Exp, relative expression of gene

\section{Introduction}

Pre-harvest sprouting (PHS) is one of the major adverse effects of high yield and stable yield of wheat (Xiao et al., 2002a; Li et al., 2004). In China, the phenomenon of wheat spike germination is particularly serious. Spike germination occurred frequently and seriously in the world's major wheat producers, including Canada, the United States, Britain, Australia, Brazil, Germany, Sweden and so on. Direct annual losses caused by PHS approach $\$ 1$ billion dollars worldwide (Liu et al., 2013). The yield reduction caused by the problem of wheat spike germination accounts for nearly $83 \%$ of the total wheat planting area. Seed viability and hydrolysis of starch and protein in the endosperm are always reduced after PHS (Xiao et al., 2002b). At present, most of the wheat varieties used in the production have certain ear sprouting characteristics. The way to solve the PHS was as follows: firstly, the application of chemical control technology; secondly, breeding varieties with resistance to PHS. Breeding resistant cultivars is the best way to solve the problem of sprouting, but the selection and application was restricted by limited resistance source. It is very important to excavate 
good genes of PHS resistance, which is an ideal way to solve the PHS problem. In order to make full use of the RING finger gene to improve the resistance to PHS in wheat, a RING finger transcription factor gene TaRHA2b was cloned from wheat by RT-PCR and RACE amplification. Bioinformatic analysis and expression profile analysis of the TaRHA2b gene with the treatment of different abscisic acid (ABA) concentration were carried out. The research could provide some reference for studying the mechanism of TaRHA2b gene in wheat dormancy, which may be used as an excellent gene resource to improve PHS resistance.

\section{Review of literature}

The ubiquitination process is accomplished by the continuous action of ubiquitinactivating enzymes (E1), ubiquitin-conjugating enzymes (E2) and ubiquitin protein ligases (E3), and the specificity of the ubiquitin-conjugating enzyme E3 binding substrate to a great extent (Smalle and Vierstra, 2004). In the Arabidopsis genome, more than 1300 genes are predicted to encode different types of E3 ligases, which means that ubiquitination is involved in a wide range of cellular metabolic processes and fine adjustment of life activities (Li et al., 2017). It was found that ubiquitination linked many components of plant biology through molecular genetic analysis, including cell cycle, embryonic development, light morphogenesis, regulation of day and night patterns, hormone signaling, homologous transformation, resistance to disease and aging (Liu et al., 2017).

Ubiquitin E3 ligases are mainly classified into three groups: RING (Really Interesting New Gene) finger protein family, HECT protein family and U-box family. At present, Ring finger protein family was found to be the largest among them. There were 469, 488, 399, 725, 330 and 688 possible RING finger E3 ligase family members in Arabidopsis thaliana, rice, poplar, soybean, grape and apple, respectively (Kraft et al., 2005; Du et al., 2009; Lim et al., 2010; Li et al., 2011).

RING finger protein consists of a large family of proteins, which is ubiquitous in eukaryotic organisms. The RING finger domain is a typical structural feature, and it has been shown that RING finger domain plays an important role in abiotic stress of plants (Zeba et al., 2009). The RING finger domain contains a conserved amino acid sequence: Cys-X2-Cys-Xn (9-39)-Cys-X (1-3) -His-X (2-3) -Cys/His-X2-Cys-X (4-48) -Cys-X2Cys (Cys is a cysteine residue, His is a histidine residue, $\mathrm{X}$ is an arbitrary amino acid residue) (Kraft et al., 2005). According to the number and location of $\mathrm{Zn}^{2+}$ binding residues Cys and His, the RING finger family can be divided into nine subgroups: RING-HC, RING-H2, RING-v, RING-C2, RING-D, RING-S/T, RING-G, RING-mH2 and RING-mHC (Li et al., 2011). At present, many RING finger proteins have been isolated from many plants.

In the previous study, RHA2b played an important positive role in ABA-mediated seed maturity and early germination in Arabidopsis (Li et al., 2011). In order to solve the problem of wheat PHS, scholars at home and abroad have conducted a series of studies on the mechanism of wheat ear germination and made many remarkable achievements. The apparent physical and physiological characteristics of wheat seeds, including ear morphology (Zanetti et al., 2000), the color of the seed coat (Torada and Amano, 2002; Bassoi and Flintham, 2005), seed structure and water absorption (King and von Wettstein-Knowles, 2000), seed dormancy (Andreoli et al., 2006; Hughes et al., 2010), ABA content (Gerjets et al., 2010) and $\alpha$-amylase activity (Major et al., 2001), was studied. The characteristics of genes related to PHS or seed dormancy and the 
positional cloning of QTLs (Zanetti et al., 2000; Major et al., 2001; Bassoi and Flintham, 2005; Somers et al., 2007; Chen et al., 2008) was studied. PHS resistance in wheat is a quantitative trait, and relevant quantitative trait locuses linked to PHS have been reported on almost all chromosomes (Ogbonnaya et al., 2008; Munkvold et al., 2009). From the existing research results, most researchers agree that the germination of wheat spike is the result of the interaction between genotype and environment. The dormancy characteristics of plants are closely related to their spike germination characteristics. PHS-resistant wheat germplasm resources are scarce. The VP1 and Trx genes have been used to increase the PHS resistance of wheat (Li et al., 2009; Huang et al., 2012). Although these attempts have yielded some progress in controlling seed dormancy and PHS, more work is required.

\section{Materials and methods}

The wheat variety "Zhengmai 9023" was tested. Zheng mai 9023 is an excellent wheat variety, but it is prone to sprouting. Further research will be carried out based on the transgenic operation of this variety to improve the resistance of PHS. Plant expression vector PGM-T, Escherichia coli DH5a and Taq DNA polymerase were purchased from Tiangen (Tiangen Biotech (Beijing) Co., Ltd., Beijing, China). MiniBEST Plant Genomic DNA Extraction Kit, Plant total RNA extraction reagent Trizol, PrimeScript ${ }^{\mathrm{TM}}$ RT reagent Kit (Perfect Real Time) kit, T4 DNA ligase, TranZol $^{\mathrm{TM}}$ Plant kit and SYBR Premix Ex Taq ${ }^{\mathrm{TM}}$ II (Tli RNaseH plus) were purchased from TaKaRa (TaKaRa Biotechnology (Dalian) Co., Ltd., Dalian, Japan).

\section{Extraction of total DNA and RNA and synthesis of cDNA first strand}

DNA was extracted from wheat germ with MiniBEST Plant Genomic DNA Extraction Kit. Trizol reagent was used to extract the total RNA from wheat germ. The extracted RNA was tested for quality and purity and then stored in a refrigerator at $80^{\circ} \mathrm{C}$ for reserve. PrimeScript ${ }^{\mathrm{TM}} \mathrm{RT}$ reagent Kit (Perfect Real Time) was used to synthesize cDNA from total RNA. All operations are performed according to the instructions of the kits. The cDNA was stored at $-80^{\circ} \mathrm{C}$ for later use.

\section{Cloning of TaRHA2b gene}

According to the amino acid sequence of RING finger gene AtRHA2b of Arabidopsis thaliana, GenBank EST data was searched and many expression sequences of its highly homologous origin were obtained. By software splicing, analysis and in vitro splicing, a complete cDNA sequence with RING finger structure domain was obtained from wheat database (National Center for Biotechnology information). According to splicing products, specific Primer5.0 software was used to design the primers for the experiment (Table 1), and target gene prediction and in vitro splicing were conducted.

The wheat cDNA was used as template for PCR amplification. PCR reaction system $20 \mu \mathrm{L}$ : 10x PCR Buffer $2.0 \mu \mathrm{L}, 2.5 \mathrm{mM}$ dNTPs $1.6 \mu \mathrm{L}, 10 \mathrm{mM}$ upstream and downstream primers each $0.8 \mu \mathrm{L}$, Taq enzyme $0.3 \mu \mathrm{L}$, cDNA template $1.0 \mu \mathrm{L}, \mathrm{ddH}_{2} \mathrm{O}$ complement $20 \mu \mathrm{L}$. Response procedures: $94^{\circ} \mathrm{C}$ pre degeneration $3 \mathrm{~min} ; 94^{\circ} \mathrm{C}$ modified $30 \mathrm{~s}, 50^{\circ} \mathrm{C}$ annealing $40 \mathrm{~s}, 72^{\circ} \mathrm{C}$ for $1 \mathrm{~min}, 30$ cycle; $72^{\circ} \mathrm{C}$ extension time for $10 \mathrm{~min}$.

The primer pairs P1-F and P1-R were designed according to the sequence. By using bioinformatics mosaic and RACE amplification, the unknown segment of the gene was 
cloned. The full-length cDNA sequence of wheat TaRHA2b gene was obtained by RTPCR amplification with specific primers P2-F and P2-R designed according to the above product sequences. The PCR products were recovered and connected with the pGEMT vector, and the recombinant plasmid transformed into E. coli DH5 $\alpha$ competent cells, and the positive clones were screened and sent to BGI (HuaDa Biotechnology co., ltd., China) for sequencing.

Table 1. Primers sequences of the experiment

\begin{tabular}{c|c}
\hline Primers name & Primer sequence (from 5'sequence-3'sequence) \\
\hline P1 & F: TGCCCGAGGAGGTCAAGGAG \\
P2-1 & R: CGTGATTGGATGGCTACTATACAAAGTG \\
& F: CAGGTGGTCCGGCCGAGGTCGAT \\
P2-2 & R: CCAGGCACACGATGCACGTCGCCG \\
& F: GCGGTGGCGGCCGACACAGAGA \\
P2 & R: CAAAATTGGGATTTTATTTAGCTTATTATTAG \\
& F: CGCAAACGATAGACAGGCCTG \\
P2-3 & R: GCTCGACAACTGTAAAGTCTAGGT \\
& F: GTCTCCGGCCATGGGGTTCCC \\
P3 & R: CGAGCTGTTGACATTTCAGATCCACTAGC \\
& F: GTTCCAATCTATGAGGGATACACGC \\
P4 & R: GAACCTCCACTGAGAACAACATTACC \\
& F: GGTGGATCGACCTCGGC \\
R: GCTGGGAAAACGAAAGACG
\end{tabular}

\section{Bioinformatics analysis of TaRHA2b gene}

Based on cDNA sequence, using NCBI site ORF Finder (https://www.ncbi.nlm.nih.gov/orffinder/) speculation TaRHA2b open reading frame, SingaIP TaRHA2b protein 4.1 (http://www.cbs.dtu.dk/services/SignalP/) analysis of signal peptide, TMHMM Server2.0 (http://www.cbs.dtu.dk/services/TMHMM/) to predict transmembrane regions (Krogh et al., 2001). Using SMART online server (http://smart.embl-heidelberg.de/) domain analysis of protein structure. The consistency of TaRHA2b homologous genes with other species was analyzed using DNAMAN, and the evolution tree was constructed with the software MEGA 7.0. The RHA2b interacting proteins network can be predicted by string software.

\section{Tissue specific expression analysis of TaRHA2b gene}

The Root, stem, leaf, cob, lemma and endosperm from 25d flowering wheat plants were taken. RNA was extracted, and reverse transcription was conducted to synthesize the first strand of cDNA.

And PCR amplification was conducted with the first strand of cDNA as template. The reaction system was the same as above. Reaction conditions: $94^{\circ} \mathrm{C}$ for $3 \mathrm{~min}, 94^{\circ} \mathrm{C}$ for $30 \mathrm{~s}, 54^{\circ} \mathrm{C}$ for $30 \mathrm{~s}, 72^{\circ} \mathrm{C}$ for $40 \mathrm{~s}$, a total of 28 cycle, $72^{\circ} \mathrm{C}$ extension time for $10 \mathrm{~min}$, electrophoresis detection of PCR products.

The Actin gene products amplified by wheat Actin gene specific primers were taken as internal reference. The primers of Actin gene used in PCR reaction were P3-F and P3-R (Table 1). And the primers of TaRHA2b gene were P4-F and P4-R (Table 1). According to the PCR reaction mixture system, PCR amplification conditions as follows: $94^{\circ} \mathrm{C} 3 \mathrm{~min}, 94^{\circ} \mathrm{C}$ for $30 \mathrm{~s}, 54^{\circ} \mathrm{C}$ for $30 \mathrm{~s}, 72^{\circ} \mathrm{C}$ for $35 \mathrm{~s}$, a total of 28 cycle. $72^{\circ} \mathrm{C}$ 
for $10 \mathrm{~min}$, RT-PCR product after $1.0 \%$ agarose electrophoresis detecting camera, preservation. The experiment was repeated for three times.

\section{Analysis of TaRHA2b gene expression under ABA treatment}

To further study the ABA response of TaRHA2b gene during seed germination, qRTPCR was used to analyze the expression of TaRHA2b gene by ABA treatment at different concentrations and at different times at the same concentration. ABA solutions of different concentrations (Table 2) were used to treat wheat seeds with full and equal grain size three days after germination after disinfection. RNA was extracted with TranZol $^{\mathrm{TM}}$ Plant kit after 12 hours of sampling. PrimeScript ${ }^{\mathrm{TM}}$ RT reagent Kit (Perfect Real Time) was used to synthesize cDNA from total RNA. All operations are performed according to the instructions of the kits. The cDNA was stored at $-80^{\circ} \mathrm{C}$ for later use.

Table 2. Different content of the ABA in the present study

\begin{tabular}{c|c}
\hline Group number & ABA content $(\boldsymbol{\mu M})$ \\
\hline 1 & 0 \\
2 & 0.25 \\
3 & 0.5 \\
4 & 1.0 \\
5 & 2.0 \\
6 & 3.0 \\
7 & 4.0 \\
8 & 5.0 \\
\hline
\end{tabular}

Wheat seeds with full and equal size after disinfection were soaked with ABA solution of $0.5 \mu \mathrm{M}$, treatment without $\mathrm{ABA}$ was set for control, and samples were taken at different time (Table 3). RNA of dry seeds without soaking was extracted at the same time. PrimeScript ${ }^{\mathrm{TM}}$ RT reagent Kit (Perfect Real Time) was used to synthesize cDNA from total RNA. The cDNA was numbered and stored at $-80^{\circ} \mathrm{C}$ for later use.

Table 3. Sampling time of the experiments with the treatment of $0.5 \mu M A B A$

\begin{tabular}{c|c}
\hline Group number & Sampling time (h) \\
\hline 1 & 0 \\
2 & 12 \\
3 & 24 \\
4 & 36 \\
5 & 48 \\
\hline
\end{tabular}

Fluorescence quantitative PCR was used to analyze the TaRHA2b gene expression under ABA treatment with the primers P4-F and P4-R and internal reference Actin gene primers $\mathrm{P} 3-\mathrm{F}$ and $\mathrm{P} 3-\mathrm{R}$ (Table 1). According to the relative quantitative method to calculate: The relative expression of gene (Rel.Exp) $=2^{-\Delta \Delta \mathrm{Ct}}$, among them $-\Delta \Delta \mathrm{Ct}=$ Calibrator $\Delta \mathrm{Ct}-\Delta \mathrm{Ct}$ (the unknown sample), $\Delta \mathrm{Ct}$ (unknown sample) $=(\mathrm{Ct}$ ) internal gene - $(\mathrm{Ct})$ target gene, Calibrator $\Delta \mathrm{Ct}=(\mathrm{Ct})$ reference sample internal gene - $(\mathrm{Ct})$ reference sample target gene. The reaction system of fluorescence quantitative PCR was $20 \mu \mathrm{L}$ : SYBR Premix Ex Taq ${ }^{\mathrm{TM}} \mathrm{II}, 10 \mu \mathrm{L}$, PCR Forward Premer $(10 \mu \mathrm{M}) 0.8 \mu \mathrm{L}$, PCR Reverse Premer $(10 \mu \mathrm{M}) 0.8 \mu \mathrm{L}$, ROX Reference Dye II (50x), cDNA template $2 \mu \mathrm{L}$, $\mathrm{ddH}_{2} \mathrm{O}$ up to $20 \mu \mathrm{L}$. 


\section{Statistical analysis}

The software package SPSS 13.0 was used for statistical analysis. For comparing results of different treatments, the Tukey ANOVA test was performed. Differences were considered significant for $\mathrm{P}<0.05$.

\section{Results}

\section{Analysis of TaRHA2b gene sequence and its coding protein structure}

cDNA cloning of wheat TaRHA2b gene was synthesized using bioinformatics splicing combined with RACE amplification. First, the amino acid sequence of RING finger gene AtRHA2b was used to search for GenBank EST data, and the wheat EST (CA741783) with high homology was obtained (Fig. 1A). Secondly, specific primers P2-1-F and P2-1-R, P2-2-F and P2-2-R were designed according to the wheat EST sequence, and 5 'RACE (Fig. 1B) and 3' RACE (Fig. 1C) were respectively used to clone the unknown regions of the TaRHA2b gene. Finally, according to the above product sequence design, the specific primers P2-F and P2-R were used to amplify the full-length TaRHA2b fragment by RT-PCR (Fig. 1D), and the target sequence was obtained. The sequencing results confirmed that the length of TaRHA2b gene was 845 $\mathrm{bp}$, including the 5 '-end non-coding sequence of $79 \mathrm{bp}$, the 3 ' -end non-coding sequence of $301 \mathrm{bp}$ and the open reading frame (ORF) of $465 \mathrm{bp}$, encoding 155 amino acids (Fig. 2), the molecular weight was about $16.94 \mathrm{kD}$, and the isoelectric point was 7.77, belonging to weakly alkaline protein. This gene was named TaRHA2b. The GenBank accession number of the TaRHA2b gene is JN661690.1.

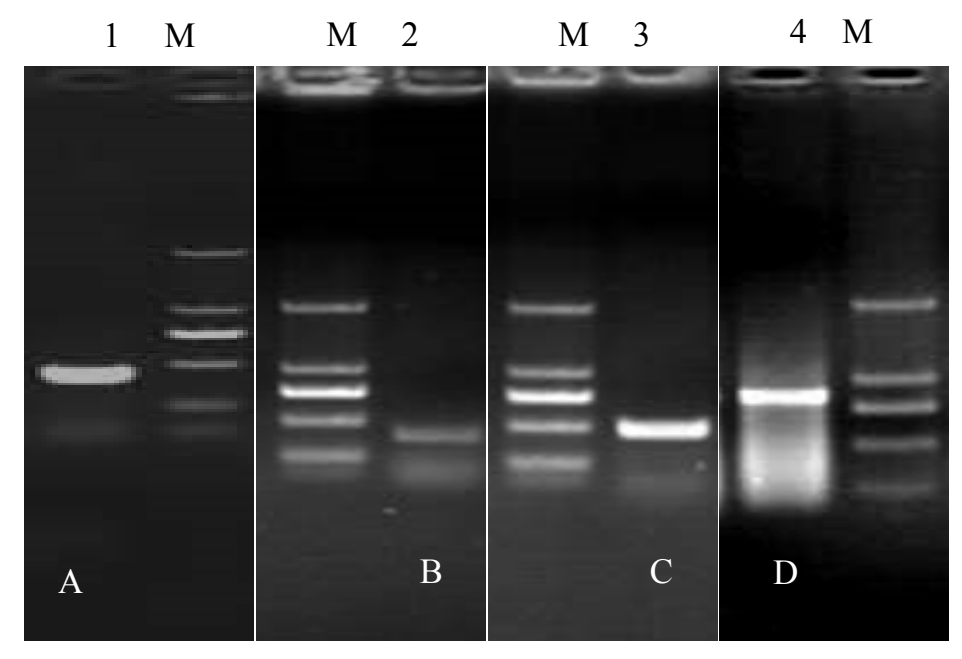

Figure 1. Amplification of $c D N A$ fragments of TaRHA2b gene in wheat immature endosperm. $A$, EST of TaRHA2b fragment; B, 3' RACE of TaRHA2b fragment; $C$, 5' RACE of TaRHA2b fragment; $D$, full-length TaRHA2b fragment; $M$, marker

To further obtain the detailed information of the TaRHA2b gene, PCR amplification was performed using primers $\mathrm{P} 2-3-\mathrm{F}$ and $\mathrm{P} 2-3-\mathrm{R}$ (Table 1) with wheat genomic DNA as the template. The amplification products were sequenced. The analysis of sequencing showed that TaRHA2b gene had no introns. 
The results of SingaIP 4.1 analysis of the signal peptide software showed that TaRHA2b contains a signal peptide sequence with a length of $32\left(\mathrm{~S}^{1}-\mathrm{F}^{32}\right)$ and $39\left(\mathrm{M}^{1}-\right.$ $\mathrm{M}^{40}$ ) amino acid residues, indicating that this kind of protein needs transmembrane transfer (localization) during the synthesis process, and then the signal peptide can be removed under the specific protease to form the mature protein sequence. Further structural analysis revealed that all RHA transcription factors, including TaRHA2b, contained motif with a length of 49 amino acid residues (RING-H2 motif), $\mathrm{CX}_{2} \mathrm{CX}_{14}$ ${ }_{15} \mathrm{CXHX}_{2} \mathrm{HX}_{2} \mathrm{CX}_{3} \mathrm{WX}_{6-8} \mathrm{CPXC}$.



Figure 2. Nucleotide and deduced amino acid sequences of the TaRHA2b gene

\section{TaRHA2b encoding amino acid sequence alignment and phylogenetic tree}

The bioinformatics software DNAMAN was used to sequence and predict the amino acid sequence of TaRHA2b gene (Fig. 2). The protein encoded by TaRHA2b gene was consisted of Zinc finger RING-type profile (Fig. 3).

\section{MGFPLVCYCVAIPKPVIAFCKLVARVDALLILISLVGLCRSPRRSVDDAPLPEEVKERLFAVEFG CLARPAQQQQHDGDDDEVAA ATCIVCLERLRATDEVRRLGHCAHAFHRGCIDGWIDLGRTTCPLC RSHLLPRARRDGPLASLLTRV"'}

\section{ZF_RING_}

Figure 3. Domain analysis of TaRHA2b protein. The yellow part of the amino acid corresponds to the blue part of the functional domain 
The sequence combination and similarity analysis of RING-H2 motif with 4 types of RING finger proteins (BOXSHADE 1.80) were performed (Fig. 4). The results showed that the amino acid sequence from wheat was similar with RHA2a and RHA2b from the Arabidopsis, indicating that this RING finger transcription factor and AtRHA2b belonged to the same family.

\begin{tabular}{|c|c|}
\hline A.RHhila & -----------------MGLPEDFTELQIFSYILKLLYIGFFRIVIALPYIG-L \\
\hline ktRHh1b & ---------------MGLPTDF-ELQIFGYVKTLYVIGFFRDMVALCPYIG-L \\
\hline A.RH $\mathrm{R} 2 \mathrm{~b}$ & 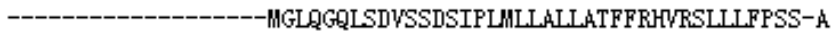 \\
\hline RsRHR2b & -------------------MGLQGQLSDVSSDSIPLMLVALLATLFKFVRSFLRFSS-S \\
\hline$k+R H k 2 a$ & ----------MGLQGQLSDVSSDSIPLMLISLLAVFIKHLRSFLLRLTSKS \\
\hline A.RHR3a & 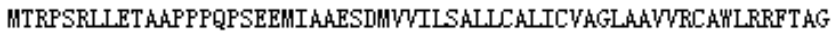 \\
\hline AtRHA3b & MTRSSRFLGT ASPPP--PEEILAAETDMVILSALUCALVCVAGLAнVARCA"NLRRLTGV \\
\hline $\mathrm{TaRH} R 2 \mathrm{~b}$ & --------------MGFPLVCCVAIPKPVIAFCKLVARVRDALLLLISLVGLCR \\
\hline \multirow[t]{2}{*}{$\mathrm{NaRHA} 4 \mathrm{a}$} & -----MGIPESPSPPHLYPQALQLKLYQAFIFSIPILFSIILFLLFYLFYLKRRASIGSI \\
\hline & : \\
\hline A.RHhl a & PRFLDHAETSAPDLTRHALSTSASLAKELIPVVRFSDLPTD----------PEDCCTVCLS \\
\hline A.RHA.b & PSFLDHATSRSDPTRLALSTSATLAHELIPVVRSDLLTD----------PEDCCTVCLS \\
\hline A.RHA2b & PVWVTS---------KLSVLADQLKLLRLFSYRYSDH------------AASDCIVCLS \\
\hline RsRHR2b & SSVVEDASLSISSGF \\
\hline$\beta+R H h 2 a$ & NPKLPVDDVSIASGLAKIIVLADQLSLRRLFSYRCGDGGG-----------GGSDCVVCLS \\
\hline A.RHh3a & 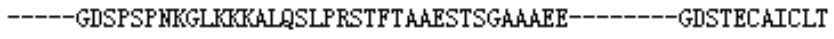 \\
\hline A.RHA3b & NPAAVGEAPPPKKGLKKKKLQALPKSTYTASASTAнAADDLPCSSVGDGDSSTECAICIT \\
\hline $\mathrm{TaRH} h 2 \mathrm{~b}$ & SPRRSVDDAPLPEEVKERLFAVEFGCLARP AQQQQHDGDDD------EVAARATCIVCLE \\
\hline HaRHA4 a & SPATVTRSSTHAIIHGEVDIKGMLKWKLPVILFDEDSMMRD------------SQCCVCLG \\
\hline A.RHhl a & DFESDDKVRQLPKCGHVFHYCLDR"IVDYKMMKCPCRHRFPKEKYTQSD"WGSGSD"WF \\
\hline AtRHA1b & DFVSDDKIRQLPKCGHVFHRCLDR"IVDCKKITCPICRHRFPEEKSTPFD"W-TSD"WF \\
\hline$\beta+R H A 2 b$ & KLKTGEEVRKL-DCRHVFHKQCLEGWLLHLA-FHCPLCRSPLLPHFHQGHGSDASISAFP \\
\hline RsRHA2b & TLKTGEEVRKL-GCRHVFHKQCLEGWLQHLW-FKCPLCRSPLVG---RGGGCESITSSFS \\
\hline AtRHA2a & KLKEGEEVRKL-ECRHVFHKKCLEGWLHQFH-FTCPLCRSALVSDDCVSKTQRSVGRDLI \\
\hline A.RHA3a & DFADGEEIRVLPLCGHSFHVECIDKNLVSRS--SCPSCRRILTPVRCDRCGHASTAEMKD \\
\hline AtRHh3b & EFSEGEEIRILPLCSHคFHVACIDKNLTSRS--SCPSCRRILVPVKDRCGHНАSTAETQ \\
\hline $\mathrm{TaRH} 2 \mathrm{~b}$ & RLRATDEVRRLGHCAHAFFRGCIDGW'IDLGR-TTCPLCRSHLLPRARRDGPLASLLTRV"' \\
\hline \multirow[t]{2}{*}{ NaRHA4a } & EFEIKEELHQLPSCKHFHVCIRHW'LRSHF--SCPLCRCHVIISRQHPQPPQQP ASHLE \\
\hline & $: \quad:::: * * * * * * * * * * *: \quad * * * *$ \\
\hline AtRHhl a & SDEVESTH-------- \\
\hline AtRHh1b & RDEVESTH--------- \\
\hline A.RHA2b & LRSTSTASSH-- \\
\hline RsRHh2b & LLSDkR------ \\
\hline AtRHh2a & SCFSLH--- \\
\hline ktRHh3a & QAHRHQHLQHSSTTIPTFLP------- \\
\hline AtRHh3b & VKDQPPHHQHPSQFTSAIIPAFLP---- \\
\hline $\mathrm{TaRH}$ h $2 \mathrm{~b}$ & \\
\hline NaRHA4a & HRHQVRLDIEEESVQRDRTIKT AKISREEQHVIIEELSSASSSSGTAEHSERDHDSWVE \\
\hline k.RHhla & ------- \\
\hline A.RHA1b & --- \\
\hline$A+R H A 2 b$ & --- \\
\hline$R \leq R H A 2 b$ & --- \\
\hline AtRHh2a & --- \\
\hline A.RHR3a & --- \\
\hline A $+R H h 3 b$ & -------- \\
\hline $\mathrm{T} a \mathrm{RH} L 2 \mathrm{~b}$ & -------- \\
\hline HaRHA4 a & TLVISIKR \\
\hline
\end{tabular}

Figure 4. Comparison of the RING finger motif sequences of four type RHA proteins. The missed base is marked with "-"; conserver residues are marked with "*”; TaRHA2b (AEQ67396), AtRHAla (NP_192876), AtRHAlb (NP_192875), AtRHA2a (NP_172962), AtRHA2b (OAP11108), AtRHA3a (NP_179337), AtRHA3b (NP_195273) and $\bar{N} a R H A 4 a$ (XP_019266728) are the RING finger motif sequences of four type RHA proteins from Triticum aestivum, Arabidopsis thaliana and Nicotiana attenuate 
By applying MAGE software, the protein sequence of RING finger transcription factor was compared multiple times to map the phylogenetic tree (Fig. 5). The results showed that TaRHA2b gene was very similar to AtRHA2b gene.

The interacting protein network of RHA2b includes KIN1(AT5G15960.1), ATDI8(AT5G66400.1), AT5G12110(AT5G12110.1), KUOX1(AT5G07480.1), KUF1(AT1G31350.1), $\quad$ AIRP1(AT4G23450.2), SDIR1(AT3G55530.1), KEG(AT5G13530.1), AT5G58410(AT5G58410.1) and NAC019(AT1G52890.1) (Fig. 6). RHA2b is involved in the ubiquitination of proteins, cell protein metabolism, and response to acidic compounds. The function of proteins interacting with RHA2b is listed in Table 4. The results showed that $R H A 2 b$ gene is essential for plant growth and development and stress response.

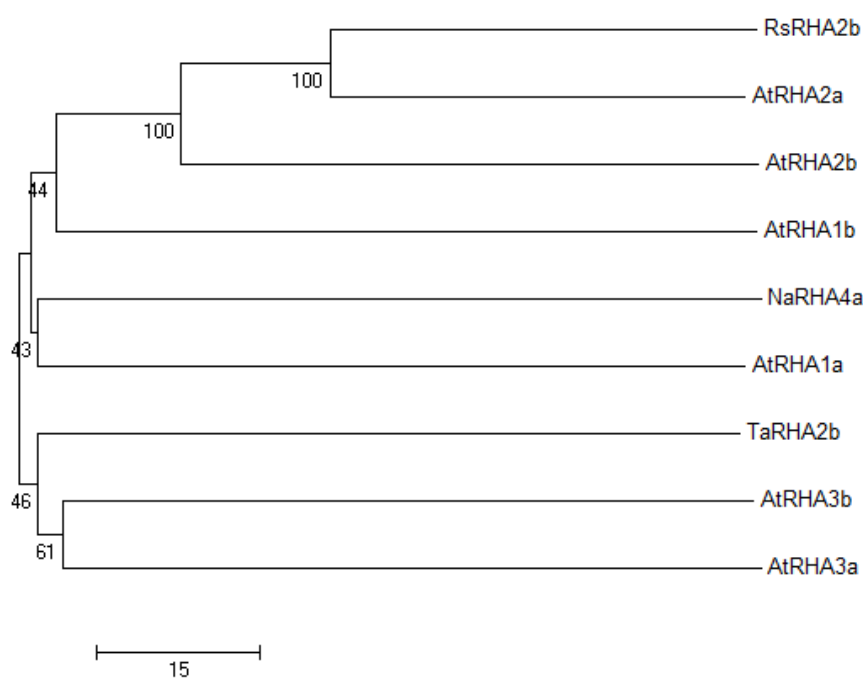

Figure 5. A phylogenetic tree of the RING finger motifs (with the software MEGA 7.0)

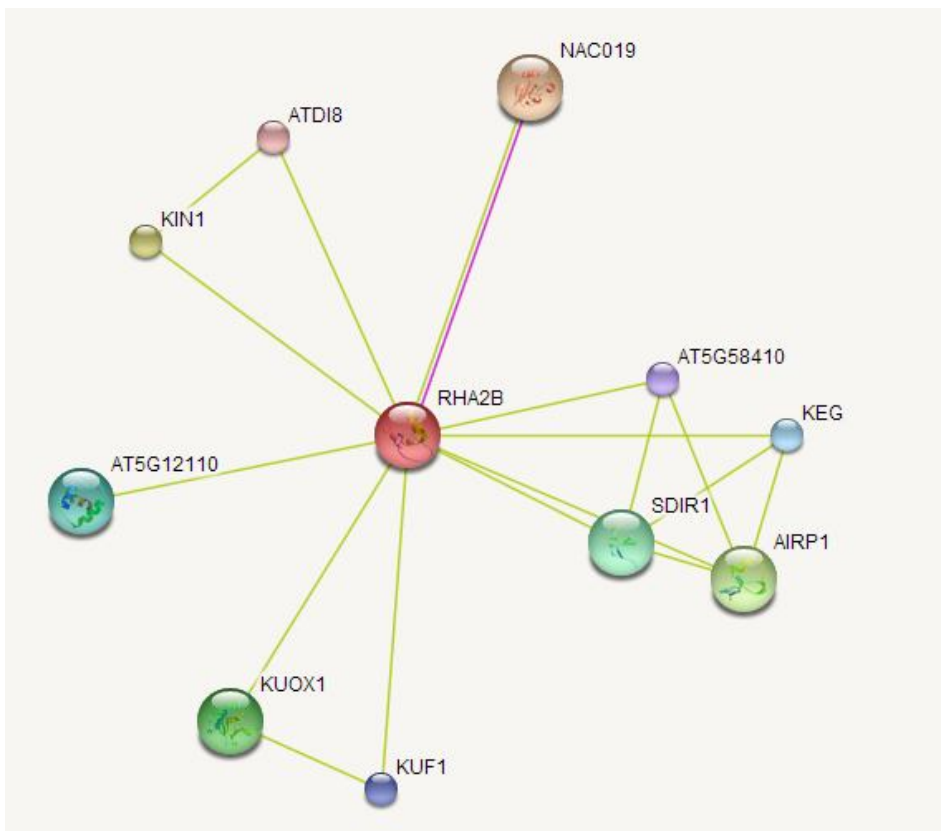

Figure 6. Interaction network of RHA2b protein (prediction by string software) 
Table 4. The function of proteins interacting with RHA2b (prediction by string software)

\begin{tabular}{c|c}
\hline Proteins & Function \\
\hline KIN1, ATDI8, SDIR1 and KEG & ABA stress response \\
KIN1, ATDI8, SDIR1 and NAC019 & water loss stress response \\
RHA2b, SDIR1, KEG, AT5G58410 and AIRP1 & the process of protein ubiquitination \\
KIN1, ATDI8, SDIR1, NAC019, RHA2b and KUF1 & non-biological stress response \\
KIN1, ATDI8, SDIR1, NAC019 and KEG & acidic and oxygen-containing stress response \\
KIN1 and ATDI8 & low temperature stress response \\
SDIR1, KEG, RHA2b, AT5G58410, AT5G12110 and KUF1 & the process of cell protein metabolism \\
\hline
\end{tabular}

\section{Tissue specific expression analysis of TaRHA2b gene}

The root, stem, leaf, cob, lemma and endosperm of wheat were used to detect expression arrays of TaRHA2b gene (Fig. 7). There was strong expression of TaRHA2b gene in the cob, lemma and endosperm tissue, while weak expression of TaRHA2b gene in root, stem and leaf tissue. The result showed that the expression of TaRHA2b gene in wheat is tissue-specific.

TaActin

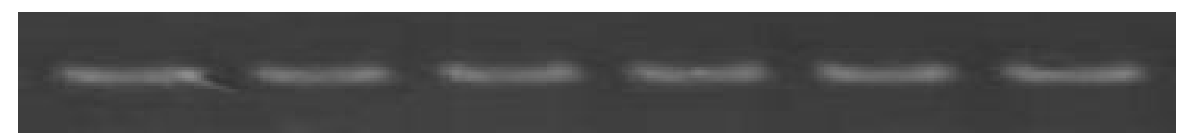

TaRHA2b

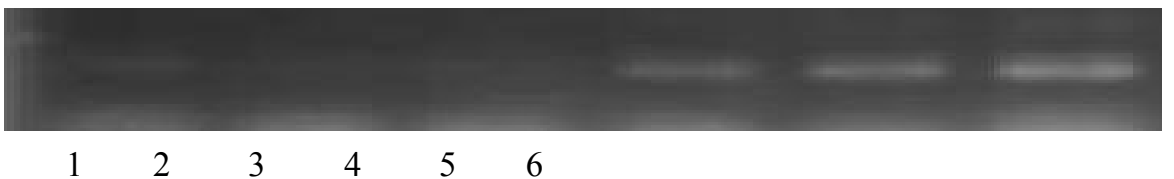

Figure 7. Expression analysis of TaRHA2b gene in different organs of wheat. Expression was analyzed by semi-quantitative RT-PCR.1, roots; 2, stems; 3, leaves; 4, cob; 5, lemma; 6, endosperm

\section{Expression profile of TaRHA2b gene under ABA treatment}

The results of tissue specific expression analysis of TaRHA2b gene showed that TaRHA2b gene was highly expressed in endosperm, and it can be inferred that TaRHA2b gene may be involved in seed germination. ABA is an important plant hormone that regulates seed maturation, development and germination. Therefore, in order to study whether TaRHA2b gene is involved in ABA signaling pathway, we analyzed the response of TaRHA2b gene to exogenous ABA during wheat seed germination.

The results showed that the transcription expression of TaRHA2b gene was induced by ABA (Fig. 8). The different ABA concentration $(0 \mu \mathrm{M}, 0.25 \mu \mathrm{M}, 0.5 \mu \mathrm{M}, 1.0 \mu \mathrm{M}$, $2.0 \mu \mathrm{M}, 3.0 \mu \mathrm{M}, 4 \mu \mathrm{M}$ and $5 \mu \mathrm{M}$ ) was used to explore whether the TaRHA2b gene was sensitive to ABA or not. With the increase of ABA concentration, the gene expression level gradually increased. The gene expression level was at the expression peak at 0.5 $\mu \mathrm{M}$, which was extremely significant compared with the control $(\mathrm{p}<0.01)$. After that, the TaRHA2b gene expression level gradually decreased. And The expression level of the TaRHA2b gene was similar between the experimental group and the control group. So the $0.5 \mu \mathrm{M}$ ABA concentration was used for follow-up research. 


\section{$A B A$ response analysis of TaRHA2b gene in early stage after germination}

It showed that TaRHA2b gene expression is highest in dry seeds (Fig. 9). During seed germination, TaRHA2b gene expression decreased as a whole compared with dry seeds. It decreased most significantly at the stage of $12 \mathrm{~h}$ with $0.5 \mu \mathrm{M}$ ABA treatment and increased at the stage of $24 \mathrm{~h}$ with $0.5 \mu \mathrm{M}$ ABA treatment. The expression of TaRHA2b gene in the samples with the treatment of $0.5 \mu \mathrm{M}$ ABA was higher than the samples without the treatment of ABA, and the second half of the S curve of TaRHA2b gene expression in the treatment group showed a tendency to be straightened.

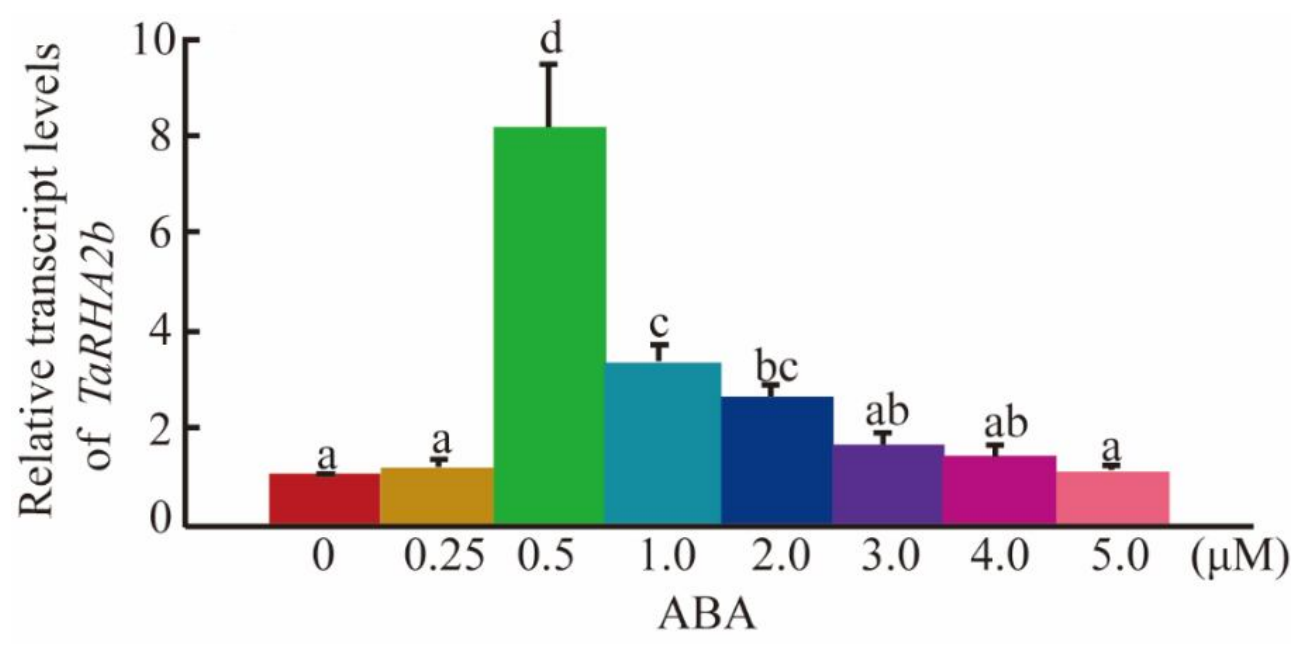

Figure 8. TaRHA2b expression in 3-d-old seedlings with the treatment of different ABA content for $12 \mathrm{~h}$. The letters ' $a$ 'to ' $d$ 'indicate statistically significant differences by Turkey's least significantly difference test $(p<0.05)$

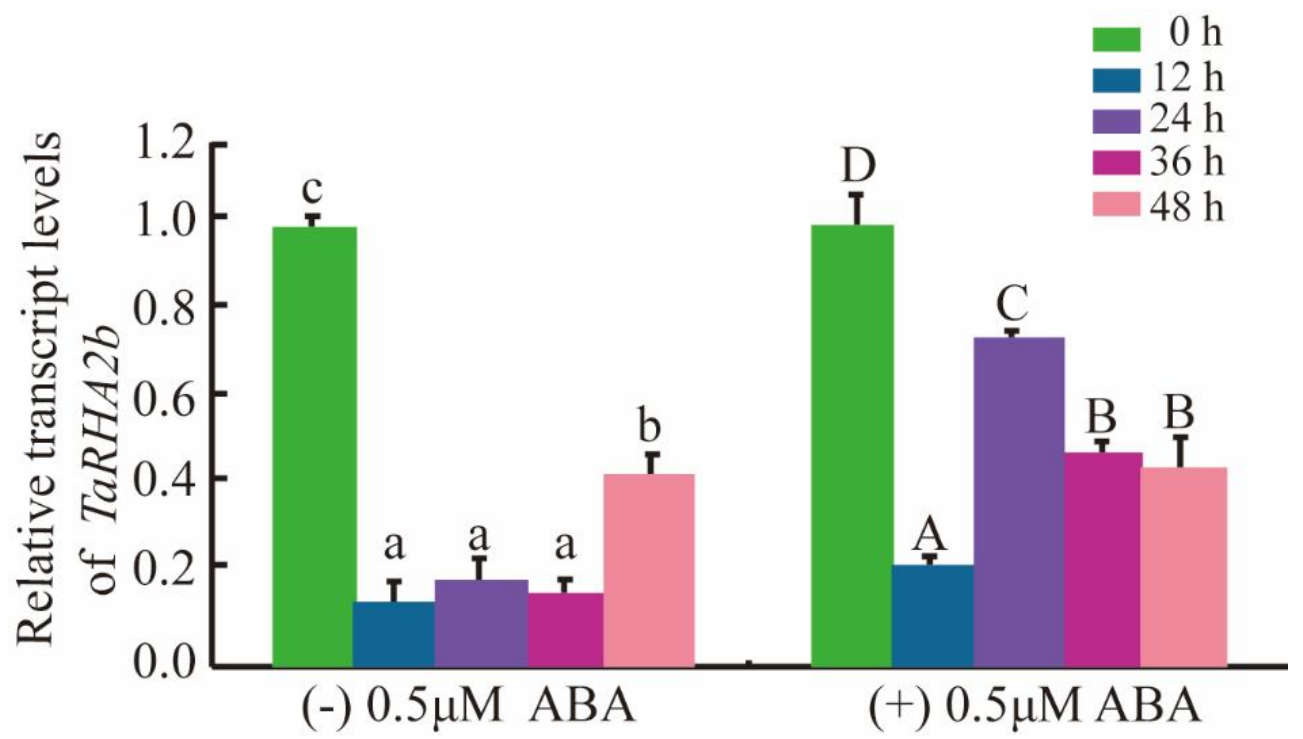

Figure 9. TaRHA2b expression in seeds during germination with the treatment of $0.5 \mu M A B A$. (-) $0.5 \mu M A B A$, the letters ' $a$ ' to ' $d$ ' indicate statistically significant differences by Turkey's least significantly difference test $(p<0.05)$; (+) $0.5 \mu M A B A$, the letters ' $A$ ' to ' $D$ ' indicate statistically significant differences by Turkey's least significantly difference test $(p<0.05)$ 


\section{Discussion}

Many studies have shown that over-expression of RING-H2 protein in model plant can affect abiotic stress (Lyzenga and Stone, 2012; Stone, 2014). Some RING finger type E3 ubiquitin ligase is involved in some specific plant signaling pathways, mainly ABA signaling pathways (Devoto and Turner, 2003; Moon et al., 2004; Schwechheimer and Schwager, 2004; Hoecker, 2005; Dreher and Callis, 2007). Several RING finger genes have been reported. AIP2 and KEG, two proteins with RING finger structure in ABA signaling pathway, are two important positive regulators of ABI3 and ABI5, respectively, which are negative regulators of ABA signaling pathway (Stone et al., 2006). It was found that CIPK26 interacted with KEG through yeast two-hybridization, and proved that CIPK26 participated in ABI5-mediated and KEG-mediated ABA stress response (Lyzenga et al., 2013). Whether there is an interaction among the three protein (CIPK26, ABI5 and KEG) is unclear. SDIRIP1, the substrate of SDIR1, was degraded by ubiquitin-proteasome pathway. SDIR1 controlled ABA related germination and stress response. SDIRIP1 participates in ABA and salt stress pathways by negatively regulating ABI5 expression (Zhang et al., 2015). Overexpression of AtXerico in Arabidopsis thaliana and rice significantly increased plant sensitivity to ABA and salt, and significantly increased plant drought resistance (Ko et al., 2006; Zeng et al., 2015). Overexpression of AtXerico homologous gene OsRHP1 in rice showed increased drought and salt tolerance, ABA content, and the expression of ABA biosynthetic genes and related genes (Zeng et al., 2014). The overexpression of ZmXerico in maize can improve drought resistance and ABA content. It is also proved that ZmXericol plays a role in ABA dynamic equilibrium by regulating the stability of ABA 8 '-hydroxylase protein and is a new control point in the ABA regulation pathway (Brugiere et al., 2017).

A RING finger transcription factor gene TaRHA2b was cloned by RT-PCR and RACE in this study. It was found that the gene belonged to the typical RING-H2 finger family. The bioinformatics analysis results showed that $T a R H A 2 b$ gene was very similar to AtRHA2b. Therefore, it is speculated that TaRHA2b is similar in function to AtRHA2b.

It can be seen that spike germination caused certain physiological damage to wheat (Fig. 10). The dormancy and germination of seeds are regulated by hormones and metabolic pathways inside the seeds. PHS resistance is closely related to seed dormancy. Varieties with strong dormancy have strong PHS resistance, while those with weak dormancy are easy to germinate. The external environment can influence the PHS resistance of wheat to some extent, but the most critical determinant is seed dormancy, especially the endogenous ABA content and the sensitivity of seeds to ABA. Genetic studies of several species have also confirmed that ABA can effectively induce seed dormancy.

Overexpression of RHA2a in transgenic Arabidopsis increased the sensitivity to ABA, increased seed dormancy and decreased seed germination rate (Bu et al., 2009). The rha2b-1 mutant with high homology between AtRHA2b and AtRHA2a also showed ABA insensitive phenotype and drought sensitivity (Li et al., 2011). The interaction between ATAF2 and RHA2a in yeast were found. RHA2a could negatively regulate Flg22-induced root growth inhibition and seedling growth inhibition in Arabidopsis thaliana and promote the growth of Pst DC3000. RHA2a can activate positive regulators by mono-ubiquitinating and stabilizing certain key regulators of the ABA signaling pathway. ANAC019 and ANAC055, two closely related NAC family proteins, were identified as RHA2a-interacting proteins (Bu et al., 2008; Jiang et al., 
2009). RHA2b targets MYB30 degradation to regulate ABA signal transduction (Zheng et al., 2018).

The analysis of the expression of wheat TaRHA2b gene showed its tissue specificity. There was higher expression of TaRHA2b gene in the cob, lemma and endosperm tissues than other tissues. It showed that TaRHA2b gene was involved in the regulation of seed formation and dormancy.

The germination rate of Arabidopsis thaliana seeds over-expression AtRHA2a and AtRHA2b significantly decreased and the sensitivity to exogenous ABA of them were enhanced. It suggested that TaRHA2b may play an equally important role in the dormancy-germination process and could be used as an excellent genetic resource to improve the germination characteristics of spike.
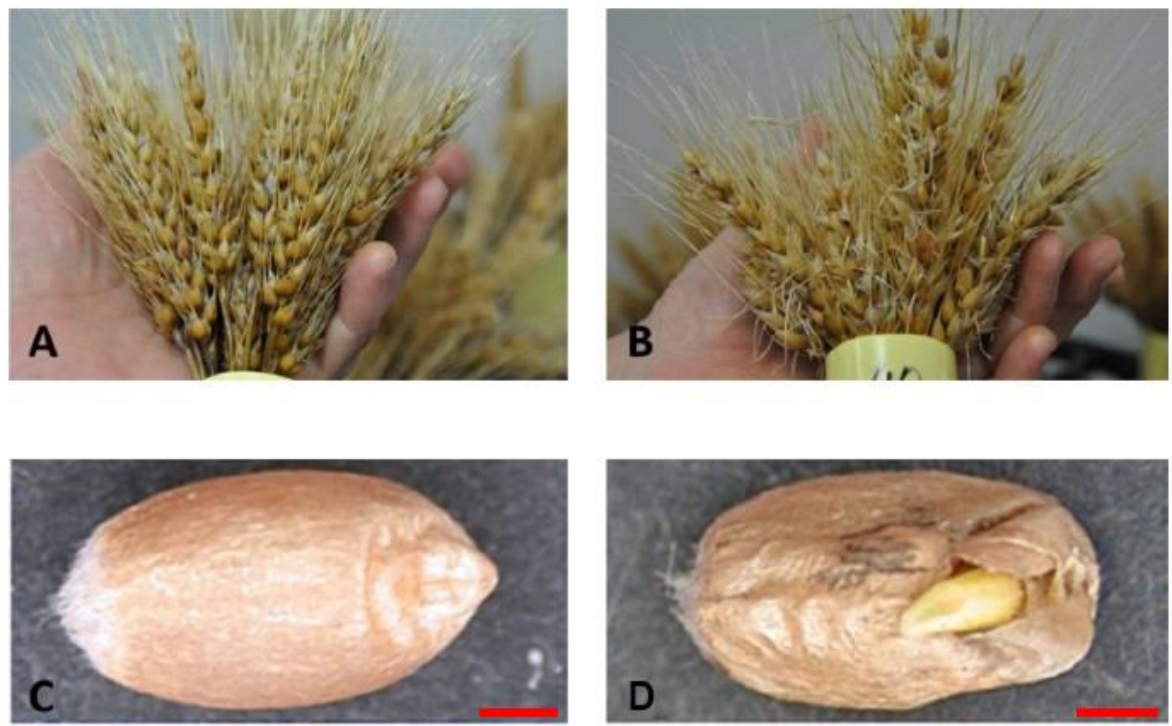

Figure 10. Physical damage of wheat caused by PHS. A: Spike of PHS-resistant germplasm; B: Spike of PHS-susceptible germplasm; C: Grain of PHS-resistant germplasm; D: Grain of PHSsusceptible germplasm. The bar value is $1 \mathrm{~mm}$

The expression profile of TaRHA2b gene with the treatment of different ABA concentration showed that TaRHA2b gene was highly expressed in dry seeds. And its expression decreased rapidly after soaking germination, which was similar to the expression pattern of similar AtRHA2b gene in Arabidopsis thaliana. The results indicated that the function of $R H A 2 b$ gene in plants is relatively similar, especially in ABA signal transduction pathway. The results showed that TaRHA2b gene may be an excellent genetic resource to improve PHS resistance. More work is needed for using TaRHA2b gene to improve dormancy and PHS tolerance in wheat.

\section{Conclusion}

The full-length cDNA of the RING finger gene TaRHA2b was cloned from wheat. The full-length cDNA of TaRHA2b encoded 154 amino acid residues, which was consisted of Zinc finger RING-type profile. The genomic TaRHA2b gene had no introns. TaRHA2b shared the identity with RHA2b from the Arabidopsis. The main result are as 
follows: (1) the expression of TaRHA2b gene was significantly tissue-specific; (1) the expression of TaRHA2b in the seeds was significantly higher than the expression of gene expression after soaking germination; (3) the sensitivity of the TaRHA2b gene to ABA was significantly increased. Some questions are as follows: (1) the distribution of TaRHA2b gene coding region in donor ancestors and different varieties could be studied to explore the mechanism mediated with TaRHA2b gene in PHS resistance;(2) the proteins interacted with TaRHA2b could be screened by yeast two hybrid system, which may be the new genes of PHS resistance genes; (3) how does the TaRHA2b gene regulate in ABA signal transduction. Further researches on the mechanism of wheat mediated with TaRHA2b gene in PHS resistance should be futher expanded in the future.

Acknowledgements. This work was supported by the "Twelfth Five-Year" National Science and Technology Projects in Rural Areas (2013BADD04B01-02) and Henan Science and Technology Project (162102110007). Hongbin Niu designed the research; Dongbing Li and Junpeng Lyu performed the experiment; Dongbing Li drafted the manuscript; Guizhen Lyu provided ideas; Xiang Wang and Jun Yin edited the manuscript; Dongbing Li and Guizhen Lyu read and approved the final manuscript. Authors state no conflict of interest.

\section{REFERENCES}

[1] Andreoli, C., Bassoi, M. C., Brunetta, D. (2006): Genetic control of seed dormancy and pre-harvest sprouting in wheat. - Scientia Agricola 63: 564-566.

[2] Bassoi, M. C., Flintham, J. (2005): Relationship between grain colour and preharvest sprouting-resistance in wheat. - Pesquisa Agropecuária Brasileira 40: 981-988.

[3] Brugiere, N., Zhang, W., Xu, Q., Scolaro, E. J., Lu, C., Kahsay, R. Y., Kise, R., Trecker, L., Williams, R. W., Hakimi, S., Niu, X., Lafitte, R., Habben, J. E. (2017): Overexpression of ring domain E3 ligase ZmXerico1 confers drought tolerance through regulation of ABA homeostasis. - Plant Physiol 175: 1350-1369.

[4] Bu, Q., Jiang, H., Li, C.-B., Zhai, Q., Zhang, J., Wu, X., Sun, J., Xie, Q., Li, C. (2008): Role of the Arabidopsis thaliana NAC transcription factors ANAC019 and ANAC055 in regulating jasmonic acid-signaled defense responses. - Cell Research 18: 756-767.

[5] Bu, Q. Y., Li, H. M., Zhao, Q. Z., Jiang, H. L., Zhai, Q. Z., Zhang, J., Wu, X. Y., Sun, J. Q., Xie, Q., Wang, D. W., Li, C. Y. (2009): The Arabidopsis ring finger E3 ligase RHA2a Is a novel positive regulator of abscisic acid signaling during seed germination and early seedling development. - Plant Physiology 150: 463-481.

[6] Chen, C. X., Cai, S. B., Bai, G. H. (2008): A major QTL controlling seed dormancy and pre-harvest sprouting resistance on chromosome $4 \mathrm{~A}$ in a Chinese wheat landrace. Molecular Breeding 21: 351-358.

[7] Devoto, A., Turner, J. G. (2003): Regulation of jasmonate-mediated plant responses in Arabidopsis. - Annals of Botany 92: 329-337.

[8] Dreher, K., Callis, J. (2007): Ubiquitin, hormones and biotic stress in plants. - Annals of Botany 99: 787-822.

[9] Du, Z., Zhou, X., Li, L., Su, Z. (2009): plantsUPS: a database of plants' Ubiquitin Proteasome System. - BMC Genomics 10: 227.

[10] Gerjets, T., Scholefield, D., Foulkes, M. J., Lenton, J. R., Holdsworth, M. J. (2010): An analysis of dormancy, ABA responsiveness, after-ripening and pre-harvest sprouting in hexaploid wheat (Triticum aestivum L.) caryopses. - Journal of Experimental Botany 61: 597-607.

[11] Hoecker, U. (2005): Regulated proteolysis in light signaling. - Current Opinion in Plant Biology 8: 469-476. 
[12] Huang, T., Qu, B., Li, H. P., Zuo, D. Y., Zhao, Z. X., Liao, Y. C. (2012): A maize viviparous 1 gene increases seed dormancy and preharvest sprouting tolerance in transgenic wheat. - Journal of Cereal Science 55: 166-173.

[13] Hughes, K., Griffey, C., Parrish, D., Barbeau, W., Souza, E., Thomason, W. (2010): Preharvest Sprouting Tolerance in Current Soft Red Winter Wheat Cultivars. - Crop science 50: 1449-1457.

[14] Jiang, H., Li, H., Bu, Q., Li, C. (2009): The RHA2a-interacting proteins ANAC019 and ANAC055 may play a dual role in regulating ABA response and jasmonate response. Plant signaling \& behavior 4: 464-466.

[15] King, R., von Wettstein-Knowles, P. (2000): Epicuticular waxes and regulation of ear wetting and pre-harvest sprouting in barley and wheat. - Euphytica 112: 157-166.

[16] Ko, J. H., Yang, S. H., Han, K. H. (2006): Upregulation of an Arabidopsis RING-H2 gene, XERICO, confers drought tolerance through increased abscisic acid biosynthesis. Plant Journal 47: 343-355.

[17] Kraft, E., Stone, S. L., Ma, L., Su, N., Gao, Y., Lau, O. S., Deng, X. W., Callis, J. (2005): Genome analysis and functional characterization of the E2 and ring-type E3 ligase ubiquitination enzymes of Arabidopsis. - Plant Physiology 139: 1597-1611.

[18] Krogh, A., Larsson, B., Von, H. G., Sonnhammer, E. L. (2001): Predicting transmembrane protein topology with a hidden Markov model: application to complete genomes. - Journal of Molecular Biology 305: 567-580.

[19] Li, C., Ni, P., Francki, M., Hunter, A., Zhang, Y., Schibeci, D., Li, H., Tarr, A., Wang, J., Cakir, M. (2004): Genes controlling seed dormancy and pre-harvest sprouting in a ricewheat-barley comparison. - Functional \& integrative genomics 4: 84-93.

[20] Li, Y. C., Ren, J. P., Cho, M. J., Zhou, S. M., Kim, Y. B., Guo, H. X., Wong, J. H., Niu, H. B., Kim, H. K., Morigasaki, S. (2009): The level of expression of thioredoxin is linked to fundamental properties and applications of wheat seeds. - Molecular plant 2: 430-441.

[21] Li, H., Jiang, H., Bu, Q., Zhao, Q., Sun, J., Xie, Q., Li, C. (2011): The Arabidopsis RING finger E3 ligase RHA2b acts additively with RHA2a in regulating abscisic acid signaling and drought response. - Plant Physiol 156: 550-63.

[22] Li, X., Hasegawa, Y., Lu, Y., Sato, T. (2017): Ubiquitin related enzymes and plantspecific ubiquitin ligase ATL family in tomato plants. - Plant Biotechnology 34: 71-78.

[23] Lim, S. D., Yim, W. C., Moon, J. C., Dong, S. K., Lee, B. M., Jang, C. S. (2010): A gene family encoding RING finger proteins in rice: their expansion, expression diversity, and co-expressed genes. - Plant Molecular Biology 72: 369.

[24] Liu, S., Sehgal, S. K., Li, J., Lin, M., Trick, H. N., Yu, J., Gill, B. S., Bai, G. (2013): Cloning and characterization of a critical regulator for preharvest sprouting in wheat. Genetics 195: 263-273.

[25] Liu, H., Ravichandran, S., Teh, O. K., Mcvey, S., Lilley, C., Teresinski, H. J., GonzalezFerrer, C., Mullen, R. T., Hofius, D., Prithiviraj, B., Stone, S. L. (2017): The ring-type E3 ligase XBAT35.2 is involved in cell death induction and pathogen response. - Plant Physiol 175: 1469-1483.

[26] Lyzenga, W. J., Stone, S. L. (2012): Abiotic stress tolerance mediated by protein ubiquitination. - Journal of Experimental Botany 63: 599-616.

[27] Lyzenga, W. J., Liu, H., Schofield, A., Muisehennessey, A., Stone, S. L. (2013): Arabidopsis CIPK26 interacts with KEG, components of the ABA signalling network and is degraded by the ubiquitin-proteasome system. - Journal of Experimental Botany 64: 2779-2791.

[28] Major, B., Kettlewell, P., Scott, R. (2001): Mechanisms leading to excess alpha-amylase activity in wheat (Triticum aestivum, L) grain in the UK. - Journal of Cereal Science 33: 313-329.

[29] Moon, R. T., Kohn, A. D., De Ferrari, G. V., Kaykas, A. (2004): WNT and beta-catenin signalling: diseases and therapies. - Nature Reviews Genetics 5: 691-701. 
[30] Munkvold, J. D., Tanaka, J., Benscher, D., Sorrells, M. E. (2009): Mapping quantitative trait loci for preharvest sprouting resistance in white wheat. - Theoretical and applied genetics 119: 1223-1235.

[31] Ogbonnaya, F. C., Imtiaz, M., Ye, G., Hearnden, P. R., Hernandez, E., Eastwood, R. F., van Ginkel, M., Shorter, S., Winchester, J. (2008): Genetic and QTL analyses of seed dormancy and preharvest sprouting resistance in the wheat germplasm CN10955. Theoretical and Applied Genetics 116: 891-902.

[32] Schwechheimer, C., Schwager, K. (2004): Regulated proteolysis and plant development. - Plant Cell Reports 23: 353-364.

[33] Smalle, J., Vierstra, R. D. (2004): Ubiquitin 26S proteasome proteolytic pathway. Annual Review of Plant Biology 55: 555-590.

[34] Somers, D., Ogbonnaya, F. C., Imtiaz, M., Depauw, R. M. (2007): Haplotype diversity of preharvest sprouting QTLs in wheat. - Genome 50: 107-118.

[35] Stone, S. L., Williams, L. A., Farmer, L. M., Vierstra, R. D., Callis, J. (2006): KEEP ON GOING, a RING E3 ligase essential for Arabidopsis growth and development, is involved in abscisic acid signaling. - Plant Cell 18: 3415-28.

[36] Stone, S. L. (2014): The role of ubiquitin and the 26S proteasome in plant abiotic stress signaling. - Frontiers in Plant Science 5: 135.

[37] Torada, A., Amano, Y. (2002): Effect of seed coat color on seed dormancy in different environments. - Euphytica 126: 99-105.

[38] Xiao, S.-H., Zhang, X.-Y., Yan, C.-S., Lin, H. (2002a): Germplasm improvement for preharvest sprouting resistance in Chinese white-grained wheat: an overview of the current strategy. - Euphytica 126: 35-38.

[39] Xiao, S., Zhang, X., Yan, C., Lin, H. (2002b): Germplasm improvement for preharvest sprouting resistance in Chinese white-grained wheat: An overview of the current strategy. - Euphytica 126: 35-38.

[40] Zanetti, S., Winzeler, M., Keller, M., Keller, B., Messmer, M. (2000): Genetic analysis of pre-harvest sprouting resistance in a wheat $\times$ spelt cross. - Crop science 40: 1406-1417.

[41] Zeba, N., Isbat, M., Kwon, N. J., Lee, M. O., Kim, S. R., Hong, C. B. (2009): Heatinducible $\mathrm{C} 3 \mathrm{HC} 4$ type RING zinc finger protein gene from Capsicum annuum enhances growth of transgenic tobacco. - Planta 229: 861-71.

[42] Zeng, D. E., Hou, P., Xiao, F., Liu, Y. (2014): Overexpressing a novel RING-H2 finger protein gene, OsRHP1, enhances drought and salt tolerance in rice ( Oryza sativa L.). Journal of Plant Biology 57: 357-365.

[43] Zeng, D. E., Hou, P., XIAO, F. \& LIU, Y. (2015): Overexpression of Arabidopsis XERICO gene confers enhanced drought and salt stress tolerance in rice ( Oryza Sativa L.). - Journal of Plant Biochemistry \& Biotechnology 24: 56-64.

[44] Zhang, H., Cui, F., Liu, L., Tian, M., Ning, Y., Lou, L., Shu, K., Wu, Y., Tang, S., Xie, Q. (2015): The ring finger ubiquitin E3 ligase SDIR1 targets SDIR1-INTERACTING PROTEIN1 for degradation to modulate the salt stress response and ABA signaling in Arabidopsis. - Plant Cell 27: 214-227.

[45] Zheng, Y., Chen, Z., Ma, L., Liao, C. (2018): The ubiquitin E3 ligase RHA2b promotes degradation of MYB30 in abscisic acid signaling. - Plant Physiology 178: 428-440. 